\title{
SEMBLANZA DRA. LIVIA ESCALONA
}

\author{
Luzardo A Canache C. ${ }^{1}$
}

Barquisimeto la capital musical venezolana, la ciudad crepuscular, aposento de la Divina Pastora, le dio la bienvenida el domingo 16 de junio de 1918, a una de sus hijas que con el tiempo enalteció el gentilicio larense y sobretodo barquisimetano. Fue bautizada en la Catedral de la ciudad, con el nombre de Livia Pastora Escalona Vásquez. Miembro de una familia de 7 hermanos, 5 damas y 2 caballeros, hijos de Don Publio Escalona y Auristela Vásquez. Su niñez e infancia transcurrió en su lar natal donde alcanzó la educación primaria y secundaria, en el Colegio Federal. En 1934, se trasladó a la ciudad de Caracas para estudiar medicina en la Universidad Central de Venezuela (UCV), gracias al apoyo e inspiración constante de su señora madre Doña Auristela. Siendo estudiante del quinto año de medicina, inauguraron la Maternidad Concepción Palacios (MCP) y el ingreso de los bachilleres fue sometido a concurso; se presentan 16 aspirantes y Livia Escalona alcanza el segundo puesto con 18 puntos sobre 20. Se graduó, en septiembre de 1940, de Doctor en Ciencias Médicas y su Tesis Doctoral fue "La contracción uterina"; esta tesis, sugerida y dirigida por el Profesor Pedro Antonio Gutiérrez Alfaro, es la única contribución venezolana al análisis de dicha contracción en trabajo de parto normal y alterado, basado en el conteo de la actividad uterina mediante el "tocógrafo de Vignes" (1). En esta promoción, por primera vez se gradúan 2 mujeres, ambas de un mismo estado, Lara, Escalona

${ }^{1}$ Miembro Titular SOGV. Miembro del Consejo Consultivo. Miembro Correspondiente Nacional Puesto N0 40. Academia Nacional de Medicina.

Forma de citar este artículo: Canache L. Semblanza Dra. Livia Escalona. Rev Obstet Ginecol Venez. 2021; 81(3):303-305. https://doi. org $/ 10.51288 / 00810315$

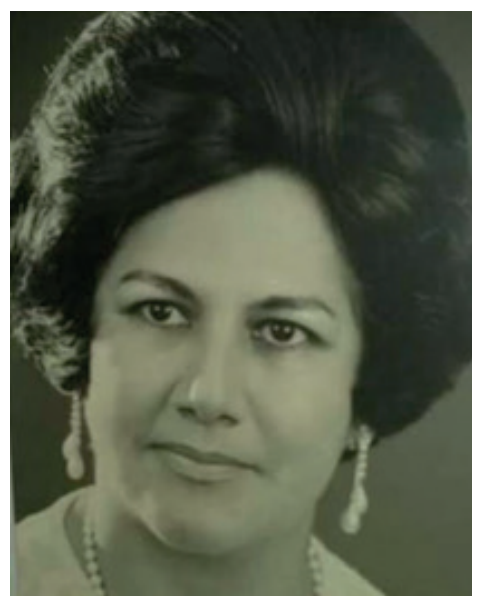

Figura 1. Dra. Livia Escalonan en 1970

Vásquez de Barquisimeto y Aura Marina Colmenares Oropeza de El Tocuyo; quien de muy niña vivió en Barquisimeto, ambas eligieron la Obstetricia.

Una vez graduada, Livia continuó sin interrupción, trabajó como adjunto del Servicio 3, dirigido por el Profesor Gutiérrez Alfaro y el otro adjunto era el Dr. Justiniano Graterol. Estuvo 7 años ad honoren, luego entró oficialmente a la nómina de MCP y se desempeñó en los Servicios 3 y 1. En 1950, se trasladó al Servicio de Esterilidad, creado por el Dr. Carlos Nouel y permaneció allí hasta 1965, cuando fue jubilada, después de 25 años ininterrumpidos en la institución (1).

Siendo estudiante y luego de graduarse, trabajó con el Dr. J T Rojas Contreras en su clínica privada y en la Cátedra de Técnica Quirúrgica. Además, figuró entre los fundadores del Colegio de Médicos del Distrito Federal. Actuó también en consulta de Ginecología del Seguro Social. 
Su consulta privada siempre la realizó en el Centro Médico de Caracas, avalada por sus maestros citados, además del gran cirujano que fue el Dr. Ricardo Baquero González. A medida que pasaban los años, se fue alejando de la Obstetricia y se dedicó a la Ginecología y Esterilidad; atendió a sus pacientes desde 1940 hasta 2002 (62 años). Su trajinar hospitalario le sirvió para figurar en publicaciones sobre sinfisiotomía, radiología y embarazo ectópico, radiopelvimetría, desprendimiento de la placenta normoinserta, hidrotubación y la tesis de grado.

La Dra. Escalona ingresó a la Sociedad de Obstetricia y Ginecología de Venezuela (SOGV), como miembro activo el 16 de octubre de 1954, con el trabajo titulado Desprendimiento prematuro de la placenta, en la "Maternidad Concepción Palacios", 1939-1950 (2), fue la segunda mujer que se incorporó a la SOGV, su compañera de grado Dra. Aura Marina Colmenares se incorporó en 1945. Inició su dilatada y fructífera trayectoria en la Sociedad. En 1957, ingresó a la Junta Directiva en calidad de suplente, participó activamente en el comité de damas de los congresos y reuniones

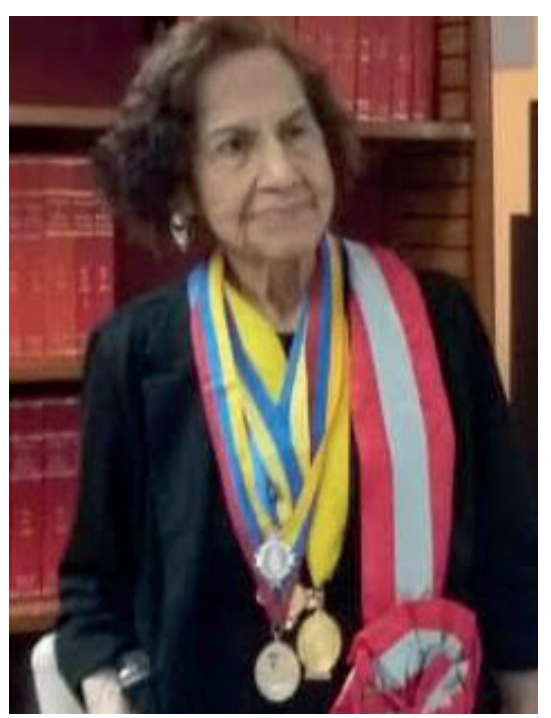

Figura 2. Dra. Livia Escalona en la celebración de su centenario nacionales de la SOGV. La entrañable amistad con el Dr. Rafael Rodríguez Navarro, fundador propietario de Laboratorios Ronava, logró que se hiciera costumbre la participación de esta institución y colaborara con los eventos de la SOGV por muchos años, así como instituciones culturales, musicales y artistas plásticos. Su labor en la SOGV, la llevó a alcanzar la presidencia, fue la primera mujer en alcanzarla, electa el 14 de febrero 1970, siendo el Vicepresidente el Dr. Héctor Martínez del Castillo, el Secretario Dr. Gustavo Pinedo Nava, Tesorero Dr. Darío Merchán López, Bibliotecario Dr. Manuel Antonio Sánchez Carvajal y los Suplentes Dres. Alfredo Díaz Bruzual, Bartolomé Azócar, Juan Bracho, Rafael Sifontes López y Antonio Vargas (3). En su gestión, buscó el acercamiento con el interior del país y de las otras especialidades, se modificó el Estatuto para incluir a los médicos cirujanos y no solo Doctores para ingresar a la SOGV, sugirió que la Junta Directiva tuviera 2 años de duración y no aceptó la reelección por enfermedad de su esposo. Al finalizar su gestión quedó en el Consejo Consultivo y trabajó intensamente tanto en la biblioteca como en la organización de los eventos de la SOGV, tanto nacionales como internacionales.

En 1974, fallece el Dr. Manuel Antonio Sánchez Carvajal, Bibliotecario de la Junta Directiva, el artífice de la Biblioteca de la SOGV, en reconocimiento a su gran labor, lleva su nombre. La Junta Directiva, presidida por el Dr. José Ignacio Páez Pumar, y el Consejo Consultivo, deciden nombrar a la Dra. Livia Escalona Bibliotecaria, por la ausencia del Dr. Sánchez Carvajal, función que desempeñó hasta 1988; fue también la primera mujer en desempeñarlo. La Junta Directiva de la Dra. Fanny V Fleitas Rodríguez, 2006-2008, reconoció su gran labor y designó la Sala de lectura Livia Escalona.

La Dra. Escalona fue una gran viajera, tanto en el aspecto científico como vacacional. En 1970, junto con el Dr. Alberto Domínguez Gallegos representaron a nuestro país en el IV Congreso Mundial de la 
Federación Internacional de Ginecología y Obstetricia (FIGO), en la ciudad de Nueva York, donde Venezuela fue designada para formar parte del Comité Ejecutivo de FIGO y el Dr. Domínguez Gallegos como Delegado Oficial de dicho comité. Su recorrido mundial por reuniones obstétricas ginecológicas y de esterilidad, merece destacar que en 1949, asistió al Primer Congreso Mexicano de la especialidad. Fue pionera en el $4^{\circ}$ Congreso Americano de 1950, en Nueva York. En 1951, asistió al Primer Convenio de Esterilidad en Rio de Janeiro y al Primer Congreso Mundial de Fertilidad en Nueva York, en 1953. Asistió a los Salones de convenciones de ciudades europeas, Ámsterdam, Berlín, Copenhague, Nápoles, Viena y en América: Bogotá, Lima, Ciudad de Panamá, San Francisco. Realizó cruceros una vez al año, visitó innumerables países, Finlandia, Rusia, Hungría, Francia, España, Portugal, Grecia.

La Dra. Escalona tuvo mucha afinidad por el arte en todas sus expresiones, la música y el canto la llevaron a fundar 3 corales, una de ellas en el Club Puerto Azul, en el litoral central, tuvo amistad con poetas, declamadores, actores y actrices, cantantes, humoristas y pintores

$\mathrm{Su}$ dilatada trayectoria la hizo merecedoras de reconocimientos, Presidente Honoraria del X Congreso Nacional de Obstetricia y Ginecología, en su ciudad natal, en 1986. Mujer FIGO, en el XV Congreso Mundial en Copenhaguen, Dinamarca, el 3 de agosto de 1997 (3). Fue Miembro Honorario de la SOGV. La Sala de Lectura de la Biblioteca Manuel Antonio Sánchez Carvajal, lleva su nombre. En 2015, la Seccional Aragua, Guárico-Apure, le hizo un reconocimiento junto con las Dras. Ofelia Uzcátegui y Tamara Samochin. También fue acreedora de distinciones, entre otras: Órdenes Mérito al Trabajo, Francisco de Miranda, Jacinto Lara, Andrés Bello y Diego de Losada. Condecoración Cruz Nacional de
Sanidad. Fue Miembro Correspondiente Extranjero de la Asociación Mexicana de Ginecología y Obstetricia y de la Sociedad Peruana de Fertilidad Matrimonial.

El maestro Agüero expresó lo siguiente: "Si Odoardo León Ponte fue calificado como el "Manolete de los parteros venezolanos" por la elegancia natural de sus actos obstétricos y quirúrgicos, Livia Escalona es la contraparte femenina, por la inteligencia y finura con la que supo evadir el asedio a que fue sometida, dados su físico y simpatía, por hombres jóvenes, maduros y hasta de la llamada tercera edad."

También manifestó que, si bien Leopoldo Aguerrevere, justificadamente, fue considerado "El Gran Señor" de la obstetricia venezolana, Livia Escalona es "La Gran Dama" de nuestra ginecoobstetricia (1)

La Dra. Escalona fue una destacada profesional, de hechura cien por ciento venezolana, una ciudadana ejemplar, honesta, de principios éticos y morales a toda prueba, que supo resolver con hidalguía los momentos desagradables que la vida le presentó, fue una mujer bondadosa, educada, elegante, distinguida, glamorosa con prestancia y sobriedad. A la Dra. Livia Escalona debemos recordarla con su sonrisa y alegría.

\section{REFERENCIAS}

1. Agüero O. Semblanza Dra. Livia Escalona. Rev Obstet Ginecol Venez.2007;67(2):135-140

2. Agüero O. Historia de la Sociedad de Obstetricia y Ginecología de Venezuela. Caracas: ATEPROCA; 2003.

3. Agüero O. Presidentes de la Sociedad de Obstetricia y Ginecología de Venezuela. Caracas: ATEPROCA; 2003.

4. Pérez D' Gregorio R, Livia Escalona. Reconocimiento de FIGO. Rev Obstet Ginecol Venez. 1997;57(3):196 